\title{
GENERICALLY TRANSITIVE ACTIONS ON MULTIPLE FLAG VARIETIES
}

\author{
ROSTISLAV DEVYATOV
}

\begin{abstract}
Let $G$ be a semisimple algebraic group whose decomposition into a product of simple components does not contain simple groups of type $A$, and $P \subseteq G$ be a parabolic subgroup. Extending the results of Popov [7, we enumerate all triples $(G, P, n)$ such that (a) there exists an open $G$-orbit on the multiple flag variety $G / P \times G / P \times \ldots \times G / P$ ( $n$ factors), (b) the number of $G$-orbits on the multiple flag variety is finite.
\end{abstract}

\section{INTRODUCTION}

Let $G$ be a semisimple connected algebraic group over an algebraically closed field of characteristic zero, and $P \subseteq G$ be a parabolic subgroup. One easily checks that the $G$-orbits on $G / P \times G / P$ are in bijection with $P$-orbits on $G / P$. The Bruhat decomposition of $G$ implies that the number of $P$-orbits on $G / P$ is finite and that these orbits are enumerated by a subset in the Weyl group $W$ corresponding to $G$. In particular, there is an open $G$-orbit on $G / P \times G / P$. So we come to the following questions: for which $G, P$ and $n \geq 3$ is there an open $G$-orbit on the multiple flag variety $(G / P)^{n}:=G / P \times G / P \times \ldots \times G / P$ ? For which $G, P$ and $n$ is the number of orbits finite?

Notice that if $G$ is locally isomorphic to $G^{(1)} \times \ldots \times G^{(k)}$, where $G^{(i)}$ are simple, then there exist parabolic subgroups $P^{(i)} \subseteq G^{(i)}$ such that $G / P \cong G^{(1)} / P^{(1)} \times \ldots \times G^{(n)} / P^{(n)}$. Hence in the sequel we may assume that $G$ is simple. Moreover, let $\pi: \widetilde{G} \rightarrow G$ be a simply connected cover. Then $\pi$ induces a bijection between parabolic subgroups $P \subseteq G$ and $\widetilde{P} \subseteq \widetilde{G}$, namely $\widetilde{P}=\pi^{-1}(P)$, and an isomorphism $\widetilde{G} / \widetilde{P} \rightarrow G / P$. Also, $\widetilde{G} / \widetilde{P}$ may be considered as $G$-variety since $\operatorname{Ker} \pi$ acts trivially on it. In this sense the isomorphism is $G$-equivariant. Therefore we may consider only one simple group of each type.

The classification of multiple flag varieties with an open $G$-orbit for maximal subgroups $P$ was given by Popov in [7]. We need some notation to formulate his result. Fix a maximal torus in $G$ and an associated simple root system $\left\{\alpha_{1}, \ldots, \alpha_{l}\right\}$ of the Lie algebra $\mathfrak{g}=$ Lie $G$. We enumerate simple roots as in [3]. Let $P_{i} \subset G$ be the maximal parabolic subgroup corresponding to the simple root $\alpha_{i}$.

Theorem 1. [7, Theorem 3] Let $G$ be a simple algebraic group. The diagonal G-action on the multiple flag variety $\left(G / P_{i}\right)^{n}$ is generically transitive if and only if $n \leq 2$ or $(G, n, i)$ is an entry in the following table:

\begin{tabular}{c|c} 
Type of $G$ & $(n, i)$ \\
\hline \hline$A_{l}$ & $n<\frac{(l+1)^{2}}{i(l+1-i)}$ \\
\hline$B_{l}, l \geq 3$ & $n=3, i=1, l$ \\
\hline$C_{l}, l \geq 2$ & $n=3, i=1, l$ \\
\hline$D_{l}, l \geq 4$ & $n=3, i=1, l-1, l$ \\
\hline$E_{6}$ & $n=3,4, i=1,6$ \\
\hline$E_{7}$ & $n=3, i=7$
\end{tabular}

In [7, the following question was posed: for which non-maximal parabolic subgroups $P \subset G$ is there an open $G$-orbit in $(G / P)^{n}$ ? We solve this problem for all simple groups except for those of type $A_{l}$. 
Denote the intersection $P_{i_{1}} \cap \ldots \cap P_{i_{s}}$ by $P_{i_{1}, \ldots, i_{s}}$. It is easy to see that $P_{i_{1}, \ldots, i_{s}}$ is a parabolic subgroup and that every parabolic subgroup is conjugated to some $P_{i_{1}, \ldots, i_{s}}$.

Theorem 2. Let $G$ be a simple algebraic group which is not locally isomorphic to $S L_{l+1}, P \subset G$ be a non-maximal parabolic subgroup and $n \geq 3$. Then the diagonal $G$-action on the multiple flag variety $\left(G / P_{i}\right)^{n}$ is generically transitive if and only if $n=3$ and $(G, P)$ is one of the following pairs:

\begin{tabular}{c|c} 
Type of $G$ & $P$ \\
\hline \hline$D_{l}, l \geq 5$ is odd & $P_{1, l-1}, P_{1, l}$ \\
\hline$D_{l}, l \geq 4$ is even & $P_{1, l-1}, P_{1, l}, P_{l-1, l}$
\end{tabular}

Now let us consider actions with a finite number of orbits. Recall that a $G$-variety $X$ is called spherical if a Borel subgroup $B \subseteq G$ acts on $X$ with an open orbit. It is well-known that the number of $B$-orbits on a spherical variety is finite, see [1, 9]. Equivalently, the number of $G$ orbits on $G / B \times X$ is finite if $X$ is spherical. Therefore, if $P \subseteq G$ is a parabolic subgroup and $X$ is a spherical $G$-variety, then the number of $G$-orbits on $G / P \times X$ is finite. The classification of all pairs of parabolic subgroups $(P, Q)$ such that $G / P \times G / Q$ is spherical is given in 4 and [8]. According to this classification, if $\left(G, P_{i}\right)$ is an entry in the table from Theorem 11, then $G / P_{i} \times G / P_{i}$ is spherical and hence the number of $G$-orbits on $G / P_{i} \times G / P_{i} \times G / P_{i}$ is finite. In the last section we prove that the number of $G$-obits on $(G / P)^{n}$ is infinite if $n \geq 4$. We also check directly that if $(G, P)$ is an entry in the table from Theorem 2, then the number of $G$-orbits on $G / P \times G / P \times G / P$ is infinite. Thus we come to the following result.

Theorem 3. Let $G$ be a simple algebraic group, $P \subset G$ be a parabolic subgroup and $n \geq 3$. The following properties are equivalent.

(1) The number of $G$-orbits on $(G / P)^{n}$ is finite.

(2) $n=3, P$ is maximal, and there is an open $G$-orbit on $G / P \times G / P \times G / P$.

(3) $n=3$, and $G / P \times G / P$ is spherical.

Corollary 1. Let $n \geq 3$. The number of $G$-orbits on $(G / P)^{n}$ is finite if and only if $n=3$ and $(G, P)$ is one of the pairs listed in the following table:

\begin{tabular}{c|c} 
Type of $G$ & $P$ \\
\hline \hline$A_{l}$ & any maximal \\
\hline$B_{l}, l \geq 2$ & $P_{1}, P_{l}$ \\
\hline$C_{l}, l \geq 3$ & $P_{1}, P_{l}$ \\
\hline$D_{l}, l \geq 4$ & $P_{1}, P_{l-1}, P_{l}$ \\
\hline$E_{6}$ & $P_{1}, P_{6}$ \\
\hline$E_{7}$ & $P_{7}$
\end{tabular}

Let us mention a more general result for classical groups. Let $Q_{(1)}, \ldots, Q_{(n)}$ be parabolic subgroups in $G$. We call the variety $G / Q_{(1)} \times \ldots \times G / Q_{(n)}$ a generalized multiple flag variety. The classification of all generalized multiple flag varieties with a finite number of $G$-orbits is given in [5] for $G=S L_{l+1}$ and in [6] for $G=S p_{2 l}$.

Proofs of Theorems 2 and 3 use methods developed in [7. The results concerning existence of an open orbit in a linear representation space in Section 2 may be of independent interest. In several cases for $G=S O_{2 l}$ the existence of an open orbit on a multiple flag variety is checked directly.

Acknowledgements. I am grateful to my scientific advisor Ivan V. Arzhantsev for posing the problem and for paying attention to my work. Also I thank professor Vladimir L. Popov for useful comments and Dmitri A. Timashev for bringing my attention to the articles [4] and [8]. 


\section{Preliminaries}

Let $G$ be a connected simple algebraic group over an algebraically closed field $\mathbb{K}$ of characteristic zero and $\mathfrak{g}=\operatorname{Lie} G$. Fix a Borel subgroup $B \subset G$ and a maximal torus $T \subset B$. These data determine a root system $\Phi$ of $\mathfrak{g}$, a positive root subsystem $\Phi^{+}$and a system of simple roots $\Delta \subseteq \Phi^{+}, \Delta=\left\{\alpha_{1}, \ldots, \alpha_{l}\right\}$. Choose a corresponding Chevalley basis $\left\{x_{i}, y_{i}, h_{i}\right\}$ of $\mathfrak{g}$. We have $\left[h, x_{i}\right]=\alpha(h) x_{i},\left[h, y_{i}\right]=-\alpha(h) y_{i}$ for all $h \in \mathfrak{t}=\operatorname{Lie} T$ and $h_{i}=\left[x_{i}, y_{i}\right]$.

Let $I=\left\{\alpha_{i_{1}}, \ldots, \alpha_{i_{s}}\right\} \subseteq \Delta$ be a subset. The Lie algebra of the parabolic subgroup $P_{I}:=$ $P_{i_{1}, \ldots, i_{s}}$ is

$$
\mathfrak{p}=\mathfrak{b} \oplus \bigoplus_{\alpha \in \Phi_{I}} \mathfrak{g}_{\alpha}
$$

where $\mathfrak{b}=$ Lie $B$ and $\Phi_{I} \subseteq \Phi^{-}$denotes the set of the negative roots such that their decomposition into the sum of simple roots does not contain the roots $\alpha_{i}, i \in I$. For example, $P_{\Delta}=B$ and $P_{\varnothing}=G$. It is known that [2, Theorem 30.1] if a parabolic group $P$ contains $B$, then $P=P_{I}$ for some $I \subseteq \Delta$. Therefore any parabolic subgroup $P \subseteq G$ is conjugate to some $P_{I}$. If $P=P_{I}$ for some $I \subseteq \Delta$, we denote by $P^{-}$the parabolic subgroup whose Lie algebra is

$$
\mathfrak{p}^{-}=\mathfrak{t} \oplus \bigoplus_{\alpha \in-\Phi_{I} \cup \Phi^{-}} \mathfrak{g}_{\alpha}
$$

Denote the weight lattice of $T$ by $\mathfrak{X}(T)$. Let $\mathfrak{X}^{+}(T)$ be the subsemigroup of dominant weights with respect to $B$. Assume first that $G$ is simply connected. Then $\mathfrak{X}^{+}$is generated by the fundamental weights $\pi_{1}, \ldots, \pi_{l}$. Given a dominant weight $\lambda$, denote the simple $G$-module with the highest weight $\lambda$ by $V(\lambda)$. If $G$ is not simply connected, we may consider a simply connected cover $p: \widetilde{G} \rightarrow G$, the dominant weight lattice $\mathfrak{X}^{+}\left(p^{-1}(T)\right)$ and the highest weight $\widetilde{G}$-module $V(\lambda)$.

Let $G$ be a simple group and $P=P_{i_{1}, \ldots, i_{s}}$ be a parabolic subgroup. Notice that if there is an open $G$-orbit on $(G / P)^{n}$, then there exists an open $G$-orbit on $\left(G / P_{i}\right)^{n}$ for all $i \in\left\{i_{1}, \ldots, i_{s}\right\}$. Indeed, since $P \subseteq P_{i}$, one has the surjective $G$-equivariant map $G / P \rightarrow G / P_{i}, g P \mapsto g P_{i}$. It induces the surjective $G$-equivariant map $\varphi:(G / P)^{n} \rightarrow\left(G / P_{i}\right)^{n}$, and the image of an open $G$-orbit on $(G / P)^{n}$ under $\varphi$ is an open $G$-orbit on $\left(G / P_{i}\right)^{n}$. Similarly, if $G$ acts on $(G / P)^{n}$ with an open orbit and $m<n$, then $G$ acts on $(G / P)^{m}$ with an open orbit.

Theorem 1 leaves us very few cases of non-maximal parabolic groups to consider. Namely, if $n>3$ and $G$ is of type $B_{l}, C_{l}$ or $D_{l}$, then $G$ never acts on $(G / P)^{n}$ with an open orbit. If $n=3$ and $G$ is of type $B_{l}$ or $C_{l}$, it suffices to consider $P=P_{1, l}$, and we show that there is no open orbit in this case. If $n=3$ and $G$ is of type $D_{l}$, an open orbit may exist only if $P=P_{I}$ where $I \subseteq\left\{\alpha_{1}, \alpha_{l-1}, \alpha_{l}\right\}$. So there are four cases to consider. We reduce the case $P_{1, l-1}$ to the case $P_{1, l}$. If $G$ is of type $E_{6}$, the only parabolic group we should consider is $P=P_{1,6}$. We show that there is no open orbit for $n=3$. If $G$ is of type $E_{7}$, if there existed an open $G$-orbit on $(G / P)^{n}$ for $n \geq 3$, then the only maximal parabolic subgroup containing $P$ would be $P_{7}$, but in this case $P$ should be maximal itself. If $G$ is of type $E_{8}, F_{4}$ or $G_{2}$, an open orbit exists for no maximal parabolic subgroups for $n \geq 3$, so there are no cases to consider.

Given a group $G$ acting on an irreducible variety $X$ with an open orbit, according to 7 we denote the maximal $n$ such that there is an open $G$-orbit on $X^{n}$ by $\operatorname{gtd}(G: X)$. If $G$ acts on $X^{n}$ with an open orbit, we say that the action $G: X$ is generically $n$-transitive.

We make use of the following fact proved by Popov.

Proposition 1. 7, Corollary 1 (ii) of Proposition 2] Let $G$ be a simple algebraic group, $P$ be a parabolic subgroup, $P^{-}$be an opposite parabolic subgroup, $L=P \cap P^{-}$be the corresponding Levi subgroup and $\mathfrak{u}^{-}$be the Lie algebra of the unipotent radical of $P^{-}$. If $P$ is conjugate to $P^{-}$, then $\operatorname{gtd}(G: G / P)=2+\operatorname{gtd}\left(L: \mathfrak{u}^{-}\right)$. 
We suppose that the group $S O_{l}$ acts in the $l$-dimensional space and preserves the bilinear form whose matrix with respect to a standard basis is

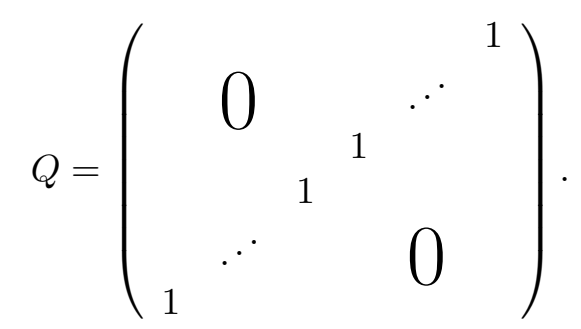

We denote the $l$-dimensional projective space by $\mathbf{P}^{l}$ and the Grassmannian of $k$-dimensional subspaces in $\mathbb{K}^{l}$ by $\operatorname{Gr}(k, l)$.

\section{EXISTENCE OF AN OPEN ORBIT}

2.1. Groups of type $B_{l}$. By Theorem 1, it is sufficient to consider the case $P=P_{1, l}$. The Dynkin diagram $B_{l}$ has no automorphisms, hence $P$ is conjugate to $P^{-}$. So we may apply Proposition 1, and it suffices to check that $\operatorname{gtd}\left(L: \mathfrak{u}^{-}\right)=0$, i. e. $L$ acts on $\mathfrak{u}^{-}$with no open orbit.

Let $G=S O_{2 l+1}$. Then $L=\mathbb{K}^{*} \times G L_{l-1}$ and the $L$-module $\mathfrak{u}^{-}$can be decomposed into the direct sum $V_{1} \oplus V_{2} \oplus V_{3} \oplus V_{4} \oplus V_{5}$. Here $V_{1}$ is a $G L_{l-1}$-module $\left(\mathbb{K}^{l-1}\right)^{*}$ dual to the tautological one and its $\mathbb{K}^{*}$-weight is $1, V_{2}$ is a trivial one-dimensional $G L_{l-1}$-module of weight $1, V_{3}$ is a tautological $G L_{l-1}$-module $\mathbb{K}^{l-1}$ of weight $0, V_{4}$ is a $G L_{l-1}$-module $\mathbb{K}^{l-1}$ of weight $1, V_{5}$ is a $G L_{l-1}$-module $\Lambda^{2} \mathbb{K}^{l-1}$ of weight 0 . According to this decomposition, we denote components of a vector $u \in \mathfrak{u}^{-}$by $u_{1}, u_{2}, u_{3}, u_{4}, u_{5}$.

Notice that there exists a $G L_{l-1}$-invariant pairing between $V_{1}$ and $V_{3}$. Its $\mathbb{K}^{*}$-weight is 1 . Also there exists a $G L_{l-1}$-invariant pairing between $V_{1}$ and $V_{4}$, whose $\mathbb{K}^{*}$-weight is 2 . Therefore the rational function

$$
\frac{\left(u_{1}, u_{3}\right)^{2}}{\left(u_{1}, u_{4}\right)}
$$

is a non-constant invariant for $L: \mathfrak{u}^{-}$, and the action of $G$ on $G / P$ is not generically 3-transitive.

2.2. Groups of type $C_{l}$. This case is completely similar to the previous one, and again the only thing we should do is to prove that there is no open $L$-orbit on $\mathfrak{u}^{-}$, where $L=P \cap P^{-}$is a Levi subgroup of $P=P_{1, l}$ and $\mathfrak{u}^{-}$is the Lie algebra of the unipotent radical of $P^{-}$.

Let $G=S p_{2 l}$. Then $L=\mathbb{K}^{*} \times G L_{l-1}$ and the $L$-module $\mathfrak{u}^{-}$can be written as $V_{1} \oplus V_{2} \oplus V_{3} \oplus V_{4}$. Here $V_{1}$ is a $G L_{l-1}$-module $\left(\mathbb{K}^{l-1}\right)^{*}$ and its $\mathbb{K}^{*}$-weight is $1, V_{2}$ is a $G L_{l-1}$-module $\mathbb{K}^{l-1}$ of weight $1, V_{3}$ is a $G L_{l-1}$-module $S^{2} \mathbb{K}^{l-1}$ of weight $0, V_{4}$ is a trivial $G L_{l-1}$-module of weight 2 . According to this decomposition, we denote components of a vector $u \in \mathfrak{u}^{-}$by $u_{1}, u_{2}, u_{3}, u_{4}$.

We see that there exists a $G L_{l-1}$-invariant pairing between $V_{1}$ and $V_{2}$ with $\mathbb{K}^{*}$-weight is 2 . Therefore we have the rational invariant

$$
\frac{\left(u_{1}, u_{2}\right)}{u_{4}}
$$

for $L: \mathfrak{u}^{-}$, and the action of $G$ on $G / P$ is not generically 3 -transitive.

2.3. Groups of type $D_{l}$. This time we should consider the following four cases of parabolic subgroups: $P=P_{1, l-1}, P_{1, l}, P_{l-1, l}, P_{1, l-1, l}$. One easily checks that $P$ and $P^{-}$are conjugate except for the cases $P=P_{1, l}, l$ odd, and $P=P_{1, l-1}, l$ odd.

Let $G=S O_{2 l}$. There exists a diagram automorphism of $G$ that interchanges $\alpha_{l-1}$ and $\alpha_{l}$. It preserves the maximal torus and the Borel subgroup and interchanges $P_{1, l-1}$ and $P_{1, l}$. Therefore, the actions $G: G / P_{1, l-1}$ and $G: G / P_{1, l}$ are either generically 3-transitive or not generically 3 -transitive simultaneously. 
2.3.1. $P=P_{l-1, l}$. In this case, $P$ and $P^{-}$are conjugate, and we have to find $\operatorname{gtd}\left(L: \mathfrak{u}^{-}\right)$.

The Levi subgroup $L$ is isomorphic to $\mathbb{K}^{*} \times G L_{l-1}$ and the $L$-module $\mathfrak{u}^{-}$is isomorphic to $V_{1} \oplus V_{2} \oplus V_{3}$, where $V_{1}$ is a $G L_{l-1}$-module $\Lambda^{2} \mathbb{K}^{l-1}$ and its $\mathbb{K}^{*}$-weight is $0, V_{2}$ is a $G L_{l-1}$-module $\mathbb{K}^{l-1}$ of weight $1, V_{3}$ is a $G L_{l-1}$-module $\mathbb{K}^{l-1}$ of weight -1 . We denote components of a vector $u \in \mathfrak{u}^{-}$by $u_{1}, u_{2}, u_{3}$.

Let $l$ be odd. Then a generic element $u_{1} \in V_{1}$ gives rise to a non-degenerate skew-symmetric bilinear form on the $G L_{l-1}$-module $\left(\mathbb{K}^{l-1}\right)^{*}$. Furthermore, one can consider the corresponding skew-symmetric form on the tautological $G L_{l-1}$-module. This form is obtained by matrix inversion and we denote it by $u_{1}^{-1}$. The following function is a rational $L$-invariant:

$$
u_{1}^{-1}\left(u_{2}, u_{3}\right) \text {. }
$$

Thus the action of $G$ on $G / P$ is not generically 3-transitive.

Let $l$ be even. We prove that there is an open $L$-orbit on $\mathfrak{u}^{-}$.

Consider the $G L_{l-1}$-module $V^{\prime}=V_{1} \oplus V_{2}$, where $V_{2}$ is a $G L_{l-1}$-module $\mathbb{K}^{l-1}$ and $V_{1}=\Lambda^{2} \mathbb{K}^{l-1}$.

Since $l-1$ is odd, the rank of a generic element $w \in V_{1}$ is $l-2$. Denote the set of all $w \in V_{1}$ such that $\operatorname{rk} w=l-2$ by $Z$. Any element $w \in Z$ gives rise to a (degenerate) skew-symmetric form on $V_{2}^{*}$, and $\operatorname{dim} \operatorname{Ker} w=1$. Consider the subspace $(\operatorname{Ker} w)^{\perp} \subset V_{2}$ where all the functions from the kernel vanish. Denote $V_{2} \backslash(\operatorname{Ker} w)^{\perp}$ by $X_{w}$. Clearly, $W_{1}=\cup_{w \in Z}\left(w \times X_{w}\right)$ is an open $G L_{l-1}$-invariant subset of $V^{\prime}$.

Let us prove that $G L_{l-1}$ acts transitively on $W_{1}$. First, given an element $u=\left(u_{1}, u_{2}\right) \in$ $W_{1}$, one can apply an element of $G L_{l-1}$ such that the matrix of the bilinear form $u_{1}$ in the corresponding basis is

$$
R=\left(\begin{array}{cccccc}
0 & & & & & \\
& 0 & 1 & & 0 & \\
& -1 & 0 & & & \\
& & & \ddots & & \\
& 0 & & & 0 & 1 \\
& & & & -1 & 0
\end{array}\right) .
$$

The first coordinate of $u_{2}$ in the new basis is non-zero since $u_{2} \in X_{u_{1}}$. Denote the $i$-th coordinate of $u_{2}$ by $\left(u_{2}\right)_{i}$. The following element of $G L_{l-1}$ preserves the bilinear form with matrix $R$ :

$$
\left(\begin{array}{cccc}
1 & 0 & \ldots & 0 \\
-\left(u_{2}\right)_{3} /\left(u_{2}\right)_{1} & & & \\
\vdots & & I_{l-2} & \\
-\left(u_{2}\right)_{l-1} /\left(u_{2}\right)_{1} & & &
\end{array}\right) .
$$

When we apply it to $u_{2}$, all its coordinates will be zero except for the first one.

So any element of $W_{1}$ can be transformed by $G L_{l-1}$-action to an element of the form $u_{1}=R$, $u_{2}=\left(\left(u_{2}\right)_{1}, 0, \ldots, 0\right)^{T}$, where $\left(u_{2}\right)_{1} \neq 0$. Clearly, all these elements belong to the same $G L_{l-1^{-}}$ orbit. Call such an element of $V^{\prime}$ canonical, i. e. call an element $\left(u_{1}, u_{2}\right) \in V^{\prime}$ canonical if $u_{1}=R, u_{2}=\left(\left(u_{2}\right)_{1}, 0, \ldots, 0\right)^{T}$, where $\left(u_{2}\right)_{1} \neq 0$. The stabilizer of $\left(u_{1},\left\langle u_{2}\right\rangle\right)$ consists of direct sums of a non-zero $1 \times 1$ matrix and a symplectic $(l-2) \times(l-2)$ matrix. Such an element fixes $u_{2}$ as well if and only if the first $1 \times 1$ matrix is 1 .

Now we are ready to consider the $L$-action on $\mathfrak{u}^{-}$. Maintain the above notation. Since $V_{2}$ and $V_{3}$ are isomorphic as $G L_{l-1}$-modules, for each $w \in Z \subset V_{1}$ we can similarly consider the open subset $V_{3} \backslash(\operatorname{Ker} w)^{\perp}$. Denote it by $Y_{w}$. Define the subsets $W_{2}=\cup_{w \in Z}\left(w \times X_{w} \times Y_{w}\right)$ and $W=\left\{u \in W_{2}: u_{2}\right.$ is not a multiple of $\left.u_{3}\right\}$. Let us prove that $L$ acts on $W$ transitively.

We may suppose that $u_{1}$ and $u_{2}$ are canonical in the sense stated above. Applying a diagonal matrix from $\left(G L_{l-1}\right)_{u_{1},\left\langle u_{2}\right\rangle}$, we may assume that $\left(u_{2}\right)_{1}\left(u_{3}\right)_{1}=1$ since $V_{2}$ and $V_{3}$ are both tautological $G L_{l-1}$-modules. Since $u_{3}$ is not a multiple of $u_{2}$, the vector $v=\left(\left(u_{3}\right)_{2},\left(u_{3}\right)_{3}, \ldots,\left(u_{3}\right)_{l-1}\right)$ is not zero. Since $S p_{l-2}$ acts transitively on $\mathbb{K}^{l-2} \backslash 0$, there exists an element $g \in\left(G L_{l-1}\right)_{u_{1}, u_{2}}$, $g=g_{1} \oplus g_{2}, g_{1}=1, g_{2} \in S p_{l-2}$ such that $g_{2} v=\left(\left(u_{3}\right)_{1}, 0, \ldots, 0\right)^{T}$. In other words, we may 
suppose that $u_{1}$ and $u_{2}$ are canonical, the only non-zero coordinates of $u_{3}$ are the first one and the second one, they are equal, and $\left(u_{2}\right)_{1}\left(u_{3}\right)_{1}=1$.

Now recall that $L=G L_{l-1} \times \mathbb{K}^{*}$, the $\mathbb{K}^{*}$-weights of $V_{1}, V_{2}$ and $V_{3}$ are 0,1 and -1 , respectively. Therefore, after applying a suitable element of $\mathbb{K}^{*}$, we have $u_{1}=S, u_{2}=(1,0, \ldots, 0)^{T}$ and $u_{3}=(1,1,0, \ldots, 0)^{T}$, and the $G$-action on $G / P$ is generically 3 -transitive.

2.3.2. $P=P_{1, l}$. In this case, Proposition 1 applies if and only if $l$ is even.

Let $l$ be even. It is sufficient to prove that there is an open $L$-orbit on $\mathfrak{u}^{-}$.

Again $L=\mathbb{K}^{*} \times G L_{l-1}$, and the $L$-module $V$ can be decomposed into three summands, $V=V_{1} \oplus V_{2} \oplus V_{3}$, but this time $V_{1}$ is a $G L_{l-1}$-module $\Lambda^{2} \mathbb{K}^{l-1}$ and its $\mathbb{K}^{*}$-weight is $0, V_{2}$ is a $G L_{l-1}$-module $\mathbb{K}^{l-1}$ of weight $1, V_{3}$ is a $G L_{l-1}$-module $\left(\mathbb{K}^{l-1}\right)^{*}$ of weight 1 . We denote components of an element $u \in \mathfrak{u}^{-}$by $u_{1}, u_{2}, u_{3}$.

Recall the notation we have introduced for the $G L_{l-1}$-module $V^{\prime}$. Also this time denote $Y_{w}=V_{3} \backslash \operatorname{Ker} w$. Define $W_{2}=\cup_{w \in Z}\left(w \times X_{w} \times Y_{w}\right)$ and $W=\left\{u \in W_{2}:\left\langle u_{2}, u_{3}\right\rangle \neq 0\right\}$. Here $\langle\cdot, \cdot\rangle$ denotes the $G L_{l-1}$-invariant pairing between $V_{2}$ and $V_{3}$. Its $\mathbb{K}^{*}$-weight is 2 , but the condition $\left\langle u_{2}, u_{3}\right\rangle \neq 0$ is not affected by $\mathbb{K}^{*}$-action, so $W$ is $L$-invariant. We are going to prove that $L$ acts transitively on $W$.

Again we may suppose that $u_{1}=R$ and the only non-zero coordinate of $u_{2}$ is the first one. Notice that $\left(u_{3}\right)_{1} \neq 0$ since $\left\langle u_{2}, u_{3}\right\rangle \neq 0$. This time $V_{2}$ and $V_{3}$ are dual $G L_{l-1}$-modules, so by applying a suitable element of $\left(G L_{l-1}\right)_{u_{1},\left\langle u_{2}\right\rangle}$ the coordinates $\left(u_{2}\right)_{1}$ and $\left(u_{3}\right)_{1}$ can be made equal.

Consider the vector $v=\left(\left(u_{3}\right)_{2},\left(u_{3}\right)_{3}, \ldots,\left(u_{3}\right)_{l-1}\right)$. It cannot be zero since $u_{3} \notin \operatorname{Ker} u_{1}$. Since $S p_{l-2}$ acts transitively on $\left(\mathbb{K}^{l-2}\right)^{*} \backslash 0$, there exists an element $g \in\left(G L_{l-1}\right)_{u_{1}, u_{2}}, g=g_{1} \oplus g_{2}$, $g_{1}=1, g_{2} \in S p_{l-2}$ such that $g_{2} v=\left(\left(u_{3}\right)_{1}, 0, \ldots, 0\right)^{T}$. In other words, we may suppose that $u_{1}$ and $u_{2}$ are canonical, the only non-zero coordinates of $u_{3}$ are the first one and the second one, and $\left(u_{2}\right)_{1}=\left(u_{3}\right)_{1}=\left(u_{3}\right)_{2}$.

This time the $\mathbb{K}^{*}$-weights of $V_{2}$ and $V_{3}$ are both 1 , so with the help of the $\mathbb{K}^{*}$-action we can satisfy the equality $\left(u_{2}\right)_{1}=\left(u_{3}\right)_{1}=\left(u_{3}\right)_{2}=1$. Thus, $L$ acts transitively on $W$, and the $G$-action on $G / P$ is generically 3 -transitive.

Let $l$ be odd. Proposition 11 does not apply, and we have to find $\operatorname{gtd}(G: G / P)$ directly.

Consider the tautological $S O_{2 l}$-module $\mathbb{K}^{2 l}$, and let $e_{1}, \ldots, e_{2 l}$ be its standard basis. Let $X^{\prime} \subset \operatorname{Gr}(l, 2 l)$ be the set of all isotropic subspaces of dimension $l$ in $\mathbb{K}^{2 l}$. One easily checks that $X^{\prime}$ is a disjoint union of two $S O_{2 l}$-orbits, and the group $O_{2 l}$ interchanges them. If two subspaces belong to the same $S O_{2 l}$-orbit, then their intersection is non-zero.

Denote the orbit $S O_{2 l}\left\langle e_{1}, \ldots, e_{l}\right\rangle \subset X^{\prime}$ by $X$. Then $X$ is an irreducible subvariety in $\operatorname{Gr}(l, 2 l)$.

For each $s \in X$ let $Y_{s} \subset \mathbf{P}^{2 l-1}$ be the set of all lines contained in $s$. Clearly, $W=\cup_{s \in X}\left(s \times Y_{s}\right)$ is a closed $G$-invariant subset in $\operatorname{Gr}(l, 2 l) \times \mathbf{P}^{2 l-1}$. One easily checks that $G / P=W$.

Let us prove that there exists an open $G$-orbit on $W \times W \times W$. We impose some conditions on the point $\left(s_{1}, a_{1}, s_{2}, a_{2}, s_{3}, a_{3}\right) \in W \times W \times W$ and so define an open subset $Y \subseteq W \times W \times W$. Then we define a point $p \in \operatorname{Gr}(l, 2 l) \times \mathbf{P}^{2 l-1} \times \operatorname{Gr}(l, 2 l) \times \mathbf{P}^{2 l-1} \times \operatorname{Gr}(l, 2 l) \times \mathbf{P}^{2 l-1}$ and prove that (a) each point $y \in Y$ belongs to the same $G$-orbit that $p$ does, and (b) $p$ belongs to $Y$. Condition (b) guarantees that $Y$ is not empty.

Let $Y \subseteq W \times W \times W$ be the set of all tuples $\left(s_{1}, a_{1}, s_{2}, a_{2}, s_{3}, a_{3}\right)$ such that:

(1) $s_{1} \cap s_{2} \cap s_{3}=0$.

(2) $s_{1}+s_{2}+s_{3}=\mathbb{K}^{2 l}$.

(3) $\operatorname{dim} s_{1} \cap s_{2}=\operatorname{dim} s_{2} \cap s_{3}=\operatorname{dim} s_{1} \cap s_{3}=1$.

(4) $\operatorname{dim}\left(a_{1}+a_{2}+a_{3}\right)=3$.

(5) The intersection of the subspaces $s=\left(s_{1} \cap s_{2}\right)+\left(s_{2} \cap s_{3}\right)+\left(s_{1} \cap s_{3}\right)$ and $a=a_{1}+a_{2}+a_{3}$ is zero.

(6) $a_{i}+s_{j}+s_{k}=\mathbb{K}^{2 l}$, where $i=1,2,3, j \neq i, k \neq i, j<k$.

(7) The lines $a_{i}$ and $a_{j}$ are not orthogonal for all $i \neq j$.

Notice that if conditions (1)-(3) hold, the sum of subspaces $s_{1} \cap s_{2}, s_{2} \cap s_{3}$ and $s_{1} \cap s_{3}$ is direct. 
Let us prove that $G$ acts transitively on $Y$. Choose vectors $f_{1}, f_{2}, f_{3}$ such that $\left\langle f_{i}\right\rangle=a_{i}$, and vectors $f_{4}, f_{5}, f_{6}$ such that $\left\langle f_{4}\right\rangle=s_{2} \cap s_{3},\left\langle f_{5}\right\rangle=s_{1} \cap s_{3},\left\langle f_{6}\right\rangle=s_{1} \cap s_{2}$. The restriction of the bilinear form to the subspace $S=\left\langle f_{1}, \ldots, f_{6}\right\rangle$ is defined by the following matrix:

$$
\left(\begin{array}{cccccc}
0 & b_{1} & b_{2} & b_{4} & & \\
b_{1} & 0 & b_{3} & & b_{5} & \\
b_{2} & b_{3} & 0 & & & b_{6} \\
b_{4} & & & & & \\
& b_{5} & & & & \\
& & b_{6} & & &
\end{array}\right) .
$$

Conditions (6) and (7) imply that $b_{i} \neq 0$ for all $i$. Clearly, this matrix is non-degenerate.

The above choice of the vectors $f_{i}$ allows to multiply them by scalars. Up to scalar multiplication we may assume that all $b_{i}=1$.

Notice that a cyclic permutation of $f_{1}, f_{2}, f_{3}$ and the same permutation of $f_{4}, f_{5}, f_{6}$ performed simultaneously define a linear operator on $S$ that preserves the restriction of the bilinear form and whose determinant is 1 .

Consider the following basis of $S: g_{1}=f_{1}, g_{2}=f_{5}, g_{3}=f_{6}, g_{4}=f_{3}-f_{4}-f_{5}, g_{5}=f_{2}-f_{4}$, $g_{6}=f_{4}$. One checks directly that the matrix of the bilinear form with respect to this basis is $Q$. Obviously, there exists a matrix $M$ such that $\left(f_{1}, \ldots, f_{6}\right)=\left(g_{1}, \ldots, g_{6}\right) M$ and whose elements do not depend on $a_{i}$ and $s_{i}$.

The restriction of the bilinear form to $S$ is non-degenerate, hence its restriction to $S^{\perp}$ is also non-degenerate. Since $s_{i}=s_{i}^{\perp}, \operatorname{dim}\left(s_{i} \cap S^{\perp}\right)=l-3$ for all $i$.

Thus, $S^{\perp}$ is a subspace of even dimension equipped with a non-degenerate symmetric bilinear form. We have three isotropic subspaces of maximal dimension in $S^{\perp}$, and the intersection of any two of them is zero. Let us prove the following lemma.

Lemma 1. Let $(\cdot, \cdot)$ be a non-degenerate symmetric bilinear form in $\mathbb{K}^{2 k}$, and $U_{1}, U_{2}, U_{3}$ be isotropic subspaces of dimension $k$ with $U_{i} \cap U_{j}=0$ for $i \neq j$. Then there exist matrices $M_{1}, M_{2}, M_{3} \in$ Mat $_{2 k \times k}$ that do not depend on $U_{i}$ and a basis $e_{1}, \ldots, e_{2 k}$ of $\mathbb{K}^{2 k}$ such that: (a) the matrix of the bilinear form is $Q$ and (b) $\left(e_{1}, \ldots, e_{2 k}\right) M_{i}$ is a basis of $U_{i}$.

Proof. Consider the non-degenerate linear map $A: U_{1} \rightarrow U_{2}$ whose graph is the subspace $U_{3}$. This is possible since $U_{i} \cap U_{j} \neq 0$ for $i \neq j$. In terms of the map, $U_{3}=\left\{v+A v \mid v \in U_{1}\right\}$.

Consider the bilinear form $\left(v_{1}, v_{2}\right)_{A}=\left(v_{1}, A v_{2}\right)$ on $U_{1}$. Since $U_{3}$ is isotropic, we have $0=$ $\left(v_{1}+A v_{1}, v_{2}+A v_{2}\right)=\left(v_{1}, v_{2}\right)+\left(A v_{1}+A v_{2}\right)+\left(v_{1}, A v_{2}\right)+\left(A v_{1}, v_{2}\right)=\left(v_{1}, A v_{2}\right)+\left(v_{2}, A v_{1}\right)=$ $\left(v_{1}, v_{2}\right)_{A}+\left(v_{2}, v_{1}\right)_{A}$ for all $v_{1}, v_{2} \in U_{1}$, hence the form $(\cdot, \cdot)_{A}$ is skew-symmetric. Assume that it is degenerate and $v \in U_{1}$ belongs to its kernel. Then $\left(v_{1}, v\right)=\left(v_{1}, A v\right)=0$ for all $v_{1} \in U_{1}$. Since the pairing between trivially intersecting isotropic subspaces $U_{1}$ and $U_{2}$ of maximal dimension is non-degenerate, $A v=0$. Since $\operatorname{Ker} A=0, v=0$ and the form $(\cdot, \cdot)_{A}$ is non-degenerate.

Thus, we have a symplectic space $U_{1}$ with the skew-symmetric form $(\cdot, \cdot)_{A}$. Hence $k$ is even. Choose a basis $\left\langle q_{1}, \ldots, q_{k}\right\rangle$ of $U_{1}$ such that the matrix of the skew-symmetric form is

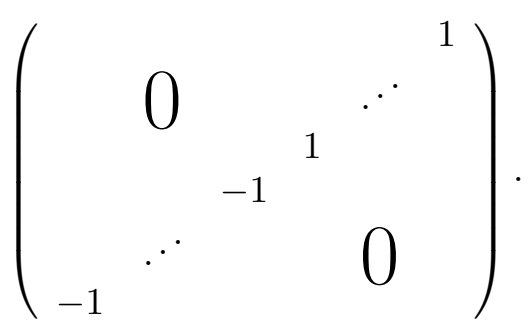

The vectors $q_{1}, \ldots, q_{k}$ are linearly independent, so let them be the first $k$ elements of a basis of $\mathbb{K}^{2 l}$. Define the rest of the basis as follows: $q_{k+j}=-A q_{j}$ if $j=1, \ldots, k / 2$ and $q_{k+j}=A q_{j}$ if $j=k / 2+1, \ldots, k$. The matrix of the bilinear form $(\cdot, \cdot)$ is $Q$. The subspaces $U_{i}$ have the 
following bases:

$$
\begin{aligned}
& U_{1}=\left\langle q_{1}, \ldots, q_{k}\right\rangle \\
& U_{2}=\left\langle q_{k+1}, \ldots, q_{2 k}\right\rangle \\
& U_{3}=\left\langle q_{1}-q_{k+1}, \ldots, q_{k / 2}-q_{k+k / 2}, q_{k / 2+1}+q_{k+k / 2+1}, \ldots, q_{k}+q_{2 k}\right\rangle .
\end{aligned}
$$

This completes the proof of the lemma.

Consider the following basis of $\mathbb{K}^{2 l}: g_{1}, g_{2}, g_{3}, q_{1}, \ldots, q_{2 l-6}, g_{4}, g_{5}, g_{6}$, where $q_{i}$ are defined above in the proof of the lemma. Notice that the matrix of the bilinear form in this basis is $Q$. Define the operator $B: \mathbb{K}^{2 l} \rightarrow \mathbb{K}^{2 l}$ that maps this basis to the standard one. We know that the matrix of the bilinear form is $Q$ in both bases, so $B \in O_{2 l}$.

Let us check that $\operatorname{det} B=1$. Assume that $\operatorname{det} B=-1$. Since $s_{1}=\left\langle g_{1}, g_{2}, g_{3}, q_{1}, \ldots, q_{l-3}\right\rangle$, $B s_{1}=\left\langle e_{1}, \ldots, e_{l}\right\rangle \in X$. Since $s_{1} \in X$, there exists an operator $C \in S O_{2 l}$ such that $C B s_{1}=s_{1}$. Thus, $C B \in\left(O_{2 l}\right)_{s_{1}}$, $\operatorname{det} C B=-1$, and the $O_{2 l}$-orbit $X^{\prime}$ cannot be a union of two distinct $\mathrm{SO}_{2 l}$-orbits, a contradiction.

Bases of the subspaces $B a_{i}$ and $B s_{i}$ can be written in terms of $e_{i}$ using matrices that do not depend on $a_{i}$ and $s_{i}$. Namely, they are the same matrices that we need to write bases of $a_{i}$ and $s_{i}$ using $g_{i}$ and $q_{i}$, and the latter do not depend on $a_{i}$ and $s_{i}$. Denote the 6-tuple $\left(B s_{1}, B a_{1}, B s_{2}, B a_{2}, B s_{3}, B a_{3}\right)$ by $p$. It suffices to prove that $p \in Y$. Conditions (1)-(7) hold by the construction of $g_{i}$ and $q_{i}$, but we should check that $\left(B s_{1}, B s_{2}, B s_{3}\right) \in X \times X \times X$. It is sufficient to find elements of $S O_{2 l}$ that map $s_{1}$ to $s_{2}$ and $s_{1}$ to $s_{3}$. Since $s_{i}=\left(s_{i} \cap S\right) \oplus\left(s_{i} \cap S^{\perp}\right)$, we find them as direct sums of elements of $S O(S)$ and $S O\left(S^{\perp}\right)$. The elements of $S O(S)$ are already found, they are cyclic permutations of $f_{1}, f_{2}, f_{3}$ and $f_{4}, f_{5}, f_{6}$. To interchange $s_{1} \cap S^{\perp}$ and $s_{2} \cap S^{\perp}$, consider the map that permutes all the pairs of vectors $g_{i} \leftrightarrow g_{2 l+1-i}, i=1, \ldots l-3$. It is orthogonal and its determinant is 1 since $l-3$ is even. Finally, the operator with the following matrix in the basis $g_{i}$ maps $s_{1} \cap S^{\perp}$ to $s_{3} \cap S^{\perp}$.

$$
\left(\begin{array}{cc}
I_{l-3} & 0 \\
D & I_{l-3}
\end{array}\right)
$$

where

$$
D=\left(\begin{array}{cccccc}
-1 & & & & & \\
& \ddots & & & 0 & \\
& & -1 & & & \\
& & & 1 & & \\
& 0 & & & \ddots & \\
& & & & & 1
\end{array}\right) .
$$

Therefore, $S O_{2 l}$ acts transitively on $Y$, and $\operatorname{gtd}(G: G / P)=3$.

2.3.3. $P=P_{1, l-1, l}$. The subgroups $P$ and $P^{-}$are conjugate for all $l$. It is sufficient to find $\operatorname{gtd}\left(L: \mathfrak{u}^{-}\right)$, where $L=\left(\mathbb{K}^{*}\right)^{2} \times G L_{l-2}$ and the $L$-module $\mathfrak{u}^{-}$is isomorphic to the direct sum of 7 simple modules that we denote by $V_{1}, \ldots, V_{7}$. Namely, $V_{1}$ is a $G L_{l-1}$-module $\left(\mathbb{K}^{l-2}\right)^{*}$ and its $\left(\mathbb{K}^{*}\right)^{2}$-weight is $(1,0), V_{2}$ is a trivial $G L_{l-2}$-module of weight $(1,1), V_{3}$ is a $G L_{l-2}$-module $\mathbb{K}^{l-2}$ of weight $(0,1), V_{4}$ is a trivial $G L_{l-2}$-module of weight $(1,-1), V_{5}$ is a $G L_{l-2}$-module $\mathbb{K}^{l-2}$ of weight $(0,-1), V_{6}$ is a $G L_{l-2}$-module $\mathbb{K}^{l-2}$ of weight $(1,0), V_{7}$ is a $G L_{l-2}$-module $\Lambda^{2} \mathbb{K}^{l-2}$ of weight $(0,0)$. Denote the components of $u \in \mathfrak{u}^{-}$by $u_{1}, \ldots, u_{7}$.

There exists a $G L_{l-2}$-invariant pairing between $V_{1}$ and $V_{3}$ whose $\left(\mathbb{K}^{*}\right)^{2}$-weight is $(1,1)$. The following function is a rational $L$-invariant:

$$
\frac{\left(u_{1}, u_{3}\right)}{u_{4}} \text {. }
$$

Thus, the $G$-action on $G / P$ is not generically 3 -transitive. 
2.4. Groups of type $E_{6}$. The only parabolic subgroup to consider is $P=P_{1,6}$. The set $\{1,6\}$ of Dynkin diagram vertices is invariant under all automorphisms of the Dynkin diagram. Hence the Weyl group element of the maximal length interchanges $P$ and $P^{-}$. We have to find $\operatorname{gtd}\left(L: \mathfrak{u}^{-}\right)$.

The Levi subgroup $L$ is locally isomorphic to $\left(\mathbb{K}^{*}\right)^{2} \times S O_{8}$, and the $L$-module $\mathfrak{u}^{-}$is isomorphic to $V_{1} \oplus V_{2} \oplus V_{3}$. Here $V_{1}$ is an $S O_{8}$-module with the lowest weight $-\pi_{1}$, i. e. a tautological $S O_{8^{-}}$ module, $\mathrm{V}_{2}$ is an $\mathrm{SO}_{8}$-module with the lowest weight $-\pi_{3}, \mathrm{~V}_{3}$ is an $\mathrm{SO}_{8}$-module with the lowest weight $-\pi_{4}$. Denote the components of $u \in \mathfrak{u}^{-}$by $u_{1}, u_{2}, u_{3}$.

Since $V_{1}$ is a tautological $\mathrm{SO}_{8}$-module, there exists an $\mathrm{SO}_{8}$-invariant symmetric bilinear form on it that we denote by $\left(u_{1}, u_{1}\right)$. There exist diagram automorphisms of $S_{8}$ that transform the tautological $\mathrm{SO}_{8}$-module to $\mathrm{SO}_{8}$-modules isomorphic to $\mathrm{V}_{2}$ and $\mathrm{V}_{3}$. So there exist an $\mathrm{SO}_{8}$ invariant on $V_{2}$ that we denote by $\left(u_{2}, u_{2}\right)$ and an $S_{8}$-invariant on $V_{3}$ that we denote by $\left(u_{3}, u_{3}\right)$. These bilinear forms are not necessarily $\left(\mathbb{K}^{*}\right)^{2}$-invariant, in general their $\left(\mathbb{K}^{*}\right)^{2}$-weights are three pairs of integers. There is a linear combination of these pairs that is equal to zero. Hence, there exists a non-trivial rational $L$-invariant of the form

$$
\left(u_{1}, u_{1}\right)^{a}\left(u_{2}, u_{2}\right)^{b}\left(u_{3}, u_{3}\right)^{c},
$$

where $a, b, c \in \mathbb{Z}$, and the $G$-action on $G / P \times G / P \times G / P$ is not generically transitive.

\section{Finite Number of orbits}

Proposition 2. Let $G$ be a simple algebraic group and $P$ be a proper parabolic subgroup. If $n \geq 4$, the number of $G$-orbits on $(G / P)^{n}$ is infinite.

Proof. Let $P=P_{i_{1}, \ldots, i_{s}}$. Consider the dominant weight $\lambda=\pi_{i_{1}}+\ldots+\pi_{i_{s}}$. Then $G / P$ is isomorphic to the projectivization of the orbit of the highest weight vector $v_{\lambda} \in V(\lambda)$. In the sequel we shortly write $i=i_{1}$. It is easy to check that $y_{i}^{2} v_{\lambda}=0$. Denote the unipotent subgroup $\exp \left(t y_{i}\right)$ by $U_{i}$. We see that $U_{i} v_{\lambda}$ is an affine line not containing zero. The closure of its image in the projectivization $\mathbf{P}(V(\lambda))$ is a projective line $\mathbf{P}^{1} \subseteq G / P \subseteq \mathbf{P}(V(\lambda))$. Choose $n \geq 4$ points $\left(x_{1}, \ldots, x_{n}\right) \in \mathbf{P}^{1} \times \ldots \times \mathbf{P}^{1} \subseteq G / P \times \ldots \times G / P$. The double ratio of the first four of these points does not change under $G$-action. Hence, two $n$-tuples with different double ratios cannot belong to the same orbit, and the number of orbits is infinite.

Now we prove that in the cases $P=P_{1, l}$ and $P=P_{l-1, l}$ the number of orbits on $G / P \times$ $G / P \times G / P$ is infinite.

We suppose that $G=S O_{2 l}$. Let $\mathbb{K}^{2 l}$ be the tautological $S O_{2 l}$-module and let $e_{1}, \ldots, e_{2 l}$ be the standard basis. Let $X^{\prime} \subset \operatorname{Gr}(l, 2 l)$ be the set of all isotropic subspaces of dimension $l$ in $\mathbb{K}^{2 l}$. It is known that $G / P_{l}$ is isomorphic to a connected component of $X^{\prime}$. In the sequel we suppose that $G / P_{l}=X \subseteq X^{\prime}$. For each $s \in X$ let $Y_{s} \subset \mathbf{P}^{2 l-1}$ be the set of all lines contained in $s$. One easily checks that the closed subset $Y=\cup_{s \in X}\left(s \times Y_{s}\right) \subset \operatorname{Gr}(l, 2 l) \times \mathbf{P}^{2 l-1}$ is isomorphic to $S O_{2 l} / P_{1, l}$.

Similarly, if $s \in X$, denote by $Z_{s} \subset \operatorname{Gr}(l-1,2 l)$ the set of all subspaces of dimension $l-1$ in $s$. Let $Z$ be the closed subset $\cup_{s \in X}\left(s \times Z_{s}\right) \subset \operatorname{Gr}(l, 2 l) \times \operatorname{Gr}(l-1,2 l)$. One easily checks that it is isomorphic to $S O_{2 l} / P_{l-1, l}$.

First, let $l=3$. Consider the following isotropic subspaces: $S_{1}=\left\langle e_{1}, e_{2}, e_{4}\right\rangle, S_{2}=\left\langle e_{2}, e_{3}, e_{6}\right\rangle$, $S_{3}=\left\langle e_{1}, e_{3}, e_{5}\right\rangle$. They belong to the same $S O_{6}$-orbit, so we may suppose that $S_{1}, S_{2}, S_{3} \in X$. Choose a line $T_{1} \subset S_{1}$ such that $T_{1} \subset\left\langle e_{1}, e_{2}\right\rangle$. Also choose lines $T_{2} \subset S_{2}$ and $T_{3} \subset S_{3}$ such that $T_{2} \subset\left\langle e_{2}, e_{3}\right\rangle$ and $T_{3} \subset\left\langle e_{1}, e_{3}\right\rangle$. Impose one more restriction, namely, the sum $T_{2}+T_{3}$ should be direct and should not be equal to $\left\langle e_{1}, e_{2}\right\rangle$. Consider the point $\left(\left(S_{1}, T_{1}\right),\left(S_{2}, T_{2}\right),\left(S_{3}, T_{3}\right)\right) \in$ $G / P_{1, l} \times G / P_{1, l} \times G / P_{1, l}$. There are four subspaces of $\left\langle e_{1}, e_{2}\right\rangle:\left\langle e_{1}\right\rangle=S_{1} \cap S_{3},\left\langle e_{2}\right\rangle=S_{2} \cap S_{3}$, $T_{1}$ and $T_{4}=\left(T_{2} \oplus T_{3}\right) \cap\left\langle e_{1}, e_{2}\right\rangle$. Thus, we have defined four lines in $\mathbb{K}^{6}$ in terms of intersections and sums of $S_{i}$ and $T_{i}$. If we apply an element $g \in G$ to these four lines, we will obtain four lines defined in the same way using $g S_{i}$ and $g T_{i}$ instead of $S_{i}$ and $T_{i}$. The double ratio of these four lines in their sum of dimension two is not changed under $G$-action. Since $T_{1}$ is chosen arbitrarily, this double ratio can be any number and the number of orbits is infinite. 
Consider the same subspaces $S_{i}$ and $T_{i}$ and set $U_{1}=T_{1} \oplus\left\langle e_{4}\right\rangle, U_{2}=T_{2} \oplus\left\langle e_{6}\right\rangle$ and $U_{3}=$ $T_{3} \oplus\left\langle e_{5}\right\rangle$. The point $\left(\left(S_{1}, U_{1}\right),\left(S_{2}, U_{2}\right),\left(S_{3}, U_{3}\right)\right)$ belongs to $Z \times Z \times Z$. Note that $\left\langle e_{1}, e_{2}, e_{3}\right\rangle=$ $\left(S_{1} \cap S_{2}\right) \oplus\left(S_{2} \cap S_{3}\right) \oplus\left(S_{1} \cap S_{3}\right)$ and $T_{i}=U_{i} \cap\left\langle e_{1}, e_{2}, e_{3}\right\rangle$. Again we have a subspace of dimension two and four lines in it defined in terms of intersections and sums of $S_{i}$ and $U_{i}$. The existence of $\mathrm{SO}_{6}$-invariant double ratio in this case yields that the number of orbits is infinite.

Let $l>3$. Construct the subspaces $S_{i}, T_{i}$ and $U_{i}$ as above, using the last three basis vectors instead of $e_{4}, e_{5}, e_{6}$. Let $S_{i}^{\prime}=S_{i} \oplus\left\langle e_{4}, \ldots, e_{l}\right\rangle$ and $U_{i}^{\prime}=U_{i} \oplus\left\langle e_{4}, \ldots, e_{l}\right\rangle$. The points $\left(\left(S_{1}^{\prime}, T_{1}\right),\left(S_{2}^{\prime}, T_{2}\right),\left(S_{3}^{\prime}, T_{3}\right)\right)$ and $\left(\left(S_{1}^{\prime}, U_{1}^{\prime}\right),\left(S_{2}^{\prime}, U_{2}^{\prime}\right),\left(S_{3}^{\prime}, U_{3}^{\prime}\right)\right)$ belong to $Y \times Y \times Y$ and $Z \times Z \times Z$, respectively. Consider also the subspace $V=\left(S_{1}^{\prime} \cap S_{2}^{\prime} \cap S_{3}^{\prime}\right)^{\perp}$. The restriction of the bilinear form to this subspace is degenerate, its kernel is $S_{1}^{\prime} \cap S_{2}^{\prime} \cap S_{3}^{\prime}=\left\langle e_{4}, \ldots, e_{l}\right\rangle$. The quotient is a space of dimension 6 with a bilinear form. The quotient morphism restricted to $\left\langle e_{1}, e_{2}, e_{3}, e_{2 l-2}, e_{2 l-1}, e_{2 l}\right\rangle$ is an isomorphism, so we have subspaces $S_{i}, T_{i}, U_{i}$ in the 6-dimensional space. This is exactly the same situation as we had above for the group $S_{6}$, and it enables us to define double ratios for the points of $G / P_{1, l} \times G / P_{1, l} \times G / P_{1, l}$ and $G / P_{l-1, l} \times G / P_{l-1, l} \times G / P_{l-1, l}$ under consideration. Therefore the number of $S O_{2 l}$-orbits on these multiple flag varieties is infinite. This finishes the proof of Theorem 3 ,

\section{REFERENCES}

[1] M. Brion, Quelques propriétés des espaces homogènes sphériques, Manuscripta Math. 55 (1986), no. 2, 191198.

[2] J. Humphreys, Linear algebraic groups. GTM 21 Springer-Verlag, New York-Heidelberg, 1975.

[3] J. Humphreys, Introduction to Lie Algebras and Representation Theory. GTM 9, Springer-Verlag, New YorkBerlin, 1978.

[4] P. Littleman, On spherical double cones, J. Algebra 166 (1994), no. 1, 142-157.

[5] P. Magyar, J. Weynman, A. Zelevinsky, Multiple flag varieties of finite type, Adv. Math. 141 (1999), no. 1, 97-118.

[6] P. Magyar, J. Weynman, A. Zelevinsky, Symplectic multiple flag varieties of finite type, J. Algebra 230 (2000), no. 1, 245-265.

[7] V.L. Popov, Generically multiple transitive algebraic group actions, Algebraic groups and homogeneous spaces, 481-523, Tata Inst. Fund. Res. Stud. Math., Tata Inst. Fund. Res., Mumbai, 2007.

[8] J. Stembridge, Multiplicity-free products and restrictions of Weyl characters., Representation Theory 7 (2003), 404-439.

[9] E.B. Vinberg, Complexity of actions of reductive groups, Funkt. Anal. i Pril. 20 (1986), no. 1, 1-13; English transl.: Funct. Anal. Appl. 20 (1986), no 1, 1-11.

Chair of Higher Algebra, Faculty of Mechanics and Mathematics, Lomonosov Moscow State University, Leninskie Gory 1, GSP-1, Moscow, 119991, Russia

E-mail address: deviatov@mccme.ru 